\title{
Multi-Parametric Families of Solutions of Order $N$ to the Boussinesq and KP Equations and the Degenerate Rational Case
}

\author{
Pierre Gaillard ${ }^{1}$ \\ ${ }^{1}$ Institut de mathématiques de Bourgogne, Université de Bourgogne, 9 avenue Alain Savary, BP 47870, 21078 Dijon Cedex, France
}

\section{Article Info}

Keywords: Boussinesq equation, Kadomtsev Petviashvili equation, Rational solutions 2010 AMS: 33Q55, 37K10 Received: 10 November 2019 Accepted: 26 March 2020 Available online: 22 June 2020

\begin{abstract}
From elementary exponential functions which depend on several parameters, we construct multi-parametric solutions to the Boussinesq equation. When we perform a passage to the limit when one of these parameters goes to 0 , we get rational solutions as a quotient of a polynomial of degree $N(N+1)-2$ in $x$ and $t$, by a polynomial of degree $N(N+1)$ in $x$ and $t$ for each positive integer $N$ depending on $3 N$ real parameters. We restrict ourself to give the explicit expressions of these rational solutions for $N=1$ until $N=3$ to shortened the paper.

We easily deduce the corresponding explicit rational solutions to the Kadomtsev Petviashvili equation for the same orders from 1 to 3.
\end{abstract}

\section{Introduction}

The Boussinesq equation in the following normalization is considered

$$
u_{t t}-u_{x x}+\left(u^{2}\right)_{x x}+\frac{1}{3} u_{x x x x}=0
$$

The subscripts $x$ and $t$ denote as usual partial derivatives.

This equation (1.1) is an equation solvable [3,4] by inverse scattering. It was introduced for the first time by Boussinesq [1,2] in 1871. This equation appears in a wide range of physical problems dealing with propagation of nonlinear waves; for example, in one-dimensional nonlinear lattice-waves [5], vibrations in a nonlinear string [6], ion sound waves in a plasma [7],...

The first solutions were constructed by Hirota [8] in 1977 with Bäcklund transformations. Non singular rational solutions were constructed by Ablowitz and Satsuma by using the Hirota bilinear method [9] in 1978. Freemann and Nimmo [10] gave in 1983 wronskians representations of the solutions. Other approaches were used; in particular, an algebro-geometrical method was given by Matveev et al. [11] in 1987; Darboux transformations [12] was used by Matveev; the $\bar{\partial}$ dressing method [13] was considered by Bogdanov.

Clarkson obtained solutions in terms of particular polynomials in a series of papers [14,15] and recently, in 2017 gives new solutions [16] as second derivatives of polynomials.

Solutions to the Boussinesq equation and the Kadomtsev Petviashvili equation are considered in this paper. We give solutions from elementary exponential functions depending on several parameters. Then we construct rational solution in performing a passage to the limit when one of these parameters goes to 0 . We obtain rational solutions as a quotient of a polynomial of degree $N(N+1)-2$ in $x$ and $t$ by a polynomial of degree $N(N+1)$ in $x$ and $t$, depending on $2 N$ parameters. We give explicit solutions in the simplest cases where $N=1,2,3$. We deduce and give explicit expressions of rational solutions to the Kadomtsev-Petviashvili (KP) equation for the cases of orders from 1 to 3. 


\section{Solutions to the Boussinesq equation}

\subsection{Solutions to the Boussinesq equation in terms of elementary exponentials}

We consider the Boussinesq equation

$$
u_{t t}-u_{x x}+\left(u^{2}\right)_{x x}+\frac{1}{3} u_{x x x x}=0 .
$$

We define the following notations.

We consider $e, a_{j}, c_{j}, d_{j}, 1 \leq j \leq N$, arbitrary real numbers, and $\alpha_{j}, \beta_{j}$ the numbers defined by

$$
\alpha_{j}=\frac{3}{2} a_{j} e+\frac{1}{2} \sqrt{1-3 a_{j}^{2} e^{2}}
$$

and

$$
\beta_{j}=-\frac{3}{2} a_{j} e+\frac{1}{2} \sqrt{1-3 a_{j}^{2} e^{2}} .
$$

We consider the following elementary functions

$$
f_{i j}(x, t)=\alpha_{j}^{i-1} \exp \left(\alpha_{j} x-\alpha_{j}^{2} t+c_{j} e^{2 N-1}\right)-\beta_{j}^{i-1} \exp \left(\beta_{j} x-\beta_{j}^{2} t+d_{j} e^{2 N-1}\right),
$$

for $1 \leq i \leq N$.

Then, we have the following statement:

Theorem 2.1. The function $v$ defined by

$$
v(x, t)=2 \partial_{x}^{2} \ln \left(\operatorname{det}\left(f_{i j}\right)_{(i, j) \in[1, N]}\right)
$$

is a solution to the Boussinesq equation (1.1) with $e, a_{j}, c_{j}$ and $d_{j}, 1 \leq j \leq N$ arbitrarily real parameters.

Proof. The corresponding Lax pair to the Boussinesq equation (1.1) is

$$
\left\{\begin{array}{l}
\phi_{x x x}+\frac{3}{2} u \phi_{x}-\frac{3}{4} \phi_{x}+u \phi=\lambda \phi, \\
\phi_{t}=-\phi_{x x}-u \phi .
\end{array}\right.
$$

The compatibility condition of the preceding system can be written as [12]

$$
\left\{\begin{array}{l}
w_{x}=\frac{3}{4} u_{x x}-\frac{3}{4} u_{t}, \\
w_{t}=\frac{1}{4} u_{x x x}+\frac{3}{4}\left(u^{2}\right)_{x}-\frac{3}{4} u_{x}+\frac{3}{4} u_{x t} .
\end{array}\right.
$$

The Boussinesq equation is obtained by excluding $w$ from the above equations.

This system is covariant by the Darboux transformation. If $\phi_{1}, \ldots, \phi_{N}$ are solutions of the system $(2.6)$, then $\phi[N]$ defined by $\phi[N]=$ $\frac{W\left(\phi_{1}, \ldots, \phi_{N}, \phi\right)}{W\left(\phi_{1}, \ldots, \phi_{N}\right)}$ is another solution of this system $(2.6)$ where $u$ is replaced by $u[N]=u+2\left(\ln W\left(\phi_{1}, \ldots, \phi_{N}\right)_{x x}[12]\right.$.

We choose $u=0$. Then the functions $\phi_{j}=f_{1 j}$ verify the following system

$$
\left\{\begin{array}{l}
\phi_{x x x}-\frac{3}{4} \phi_{x}=\lambda \phi \\
\phi_{t}=-\phi_{x x}
\end{array}\right.
$$

Then the solution of $(1.1)$ can be written as $v(x, t)=2\left(\ln W\left(\phi_{1}, \ldots, \phi_{N}\right)_{x x}\right.$ which is nothing else that $(2.4) v(x, t)=2 \partial_{x}^{2} \ln \left(\operatorname{det}\left(f_{i j}\right)_{(i, j) \in[1, N]}\right)$.

\subsection{Rational solutions to the Boussinesq equation}

To obtain rational solutions to the Boussinesq equation, we are going to perform a limit when the parameter $e$ tends to 0 .

\subsubsection{Rational solutions as a limit case}

We get the following result :

Theorem 2.2. The function $v$ defined by

$$
v(x, t)=\lim _{e \rightarrow 0} 2 \partial_{x}^{2} \ln \left(\operatorname{det}\left(f_{i j}\right)_{(i, j) \in[1, N]}\right)
$$

is a rational solution to the Boussinesq equation (1.1) depending on $3 N$ parameters $a_{j}, c_{j}$ and $d_{j}, 1 \leq j \leq N$; the numerator is a polynomial of degree $N(N+1)-2$ in $x$ and $t$, the denominator a polynomial of degree $N(N+1)$ in $x$ and $t$. 


\subsubsection{Degenerate rational solutions}

A more precise result can be formulated in the following way.

We consider $e, a_{j}, c_{j}, d_{j}, 1 \leq j \leq N$, arbitrary real numbers, and $\gamma_{j}, \delta_{j}$ the numbers defined by

$$
\begin{gathered}
\gamma_{j}=\frac{3}{2}\left(\sum_{k=1}^{N} a_{k}(j e)^{2 k-1}\right)+\frac{1}{2} \sqrt{1-3\left(\sum_{k=1}^{N} a_{k}(j e)^{2 k-1}\right)^{2}}, \\
\delta_{j}=-\frac{3}{2}\left(\sum_{k=1}^{N} a_{k}(j e)^{2 k-1}\right)+\frac{1}{2} \sqrt{1-3\left(\sum_{k=1}^{N} a_{k}(j e)^{2 k-1}\right)^{2}}
\end{gathered}
$$

We consider the following elementary functions

$$
\begin{aligned}
& \left.g_{i j}(x, t, e)=\gamma_{j}^{i-1} \exp \left(\gamma_{j} x-\gamma_{j}^{2} t+\sum_{k=1}^{N} c_{k}(j e)^{2 k-1}\right)-\delta_{j}^{i-1} \exp \left(\delta_{j} x-\delta_{j}^{2} t+\sum_{k=1}^{N} d_{k}(j e)^{2 k-1}\right)\right), \\
& \varphi_{i j}(x, t)=\frac{\partial^{j} g_{i 1}(x, t, 0)}{\partial e^{j}}, \text { for } 1 \leq i \leq N, \quad 1 \leq j \leq N .
\end{aligned}
$$

Then get the following result :

Theorem 2.3. The function $v$ defined by

$$
v(x, t)=2 \partial_{x}^{2} \ln \left(\operatorname{det}\left(\varphi_{i j}\right)_{(i, j) \in[1, N]}\right.
$$

is a rational solution to the Boussinesq equation (1.1) depending on $3 N$ parameters $a_{j}, c_{j}$ and $d_{j}, 1 \leq j \leq N$; the numerator is a polynomial of degree $N(N+1)-2$ in $x$ and $t$, the denominator a polynomial of degree $N(N+1)$ in $x$ and $t$.

Proof. In the coefficients $\alpha_{j}$ and $\beta_{j}$ defined in $(2.1,2.2)$, we replace $a_{j}$ by $\sum_{k=1}^{N} a_{k}(j e)^{2 k-1}$, and in the functions $f_{i j}$ defined in $(2.3), c_{j}$ by $\sum_{k=1}^{N} c_{k}(j e)^{2 k-1}$ and $d_{j}$ by $\sum_{k=1}^{N} d_{k}(j e)^{2 k-1}$; this gives functions $g_{i j}$ defined by (2.10). Then, it is sufficient to combine the columns of the determinant obtained from this defined by $(2.8)$ by replacing $f_{i j}$ by $g_{i j}$ and to take a passage to the limit when $e$ tends to 0 . So we get the solution $v$ given by (2.12).

So we obtain an infinite hierarchy of rational solutions to the Boussinesq equation depending on the integer $N$.

In the following we give some examples of rational solutions.

These results are consequences of the previous result (2.12).

But, it is also to possible to prove it directly in replacing the expressions of each of the solutions given in the corresponding equation and check that the relation is verified.

\subsection{First order rational solutions}

We have the following result at order $N=1$ :

Theorem 2.4. The function $v$ defined by

$$
v(x, t)=\frac{-18 a_{1}^{2}}{\left(-3 a_{1} x-c_{1}+3 t a_{1}+d_{1}\right)^{2}},
$$

is a solution to the Boussinesq equation (1.1) with $a_{1}, c_{1}, d_{1}$ arbitrarily real parameters.

Remark 2.5. If $a_{1}=0$, then the solution is the trivial solution 0 .

Remark 2.6. The solution (2.13) can be simplified and be rewritten as a solution depending on one parameter $C_{1}$.

$$
v(x, t)=\frac{-18}{\left(-3 x+3 t+C_{1}\right)^{2}} .
$$



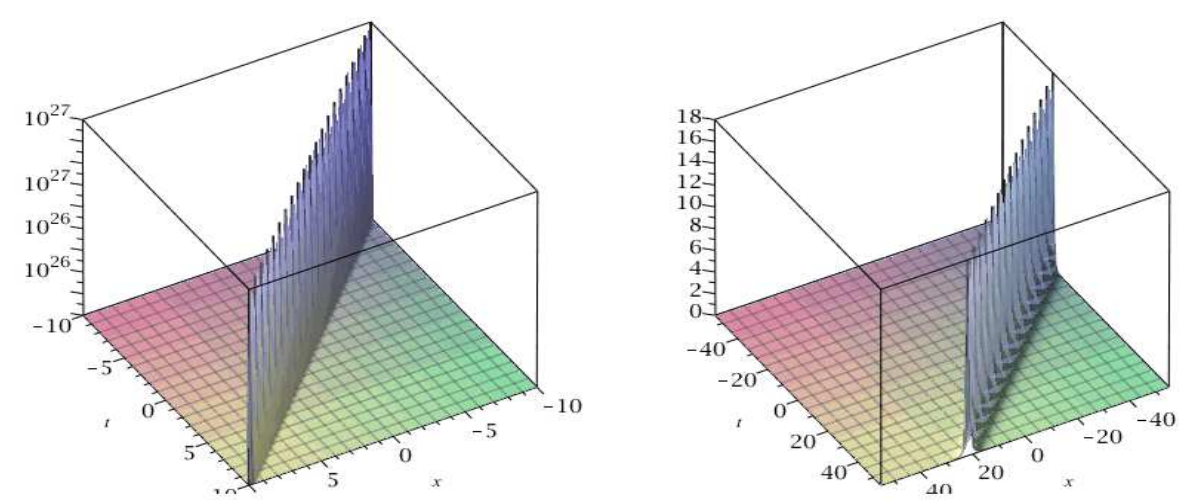

Figure 2.1: Solution of order 1 to $(1.1)$, on the left $a_{1}=10^{13}, c_{1}=1, c_{1}=0$; on the right $a_{1}=1, c_{1}=10^{2}, d_{1}=0$.

Remark 2.7. The case where $a_{1}=1, c_{1}=0, d_{1}=10^{2}$ gives the same figure as the case $a_{1}=1, c_{1}=10^{2}, d_{1}=0$. The roles played by the parameters $c$ and $d$ being the same, we only give the figures with parameters $d$ equal to 0 .

\subsection{Second order rational solutions}

Theorem 2.8. The function $v$ defined by

$$
v(x, t)=-2 \frac{n(x, t)}{d(x, t)^{2}}
$$

with

$n(x, t)=9 a_{1} a_{2}\left(27 a_{1}{ }^{5} a_{2}+243 a_{2}^{5} a_{1}-162 a_{2}{ }^{3} a_{1}^{3}\right) x^{4}+9 a_{1} a_{2}\left(-972 a_{2}{ }^{5} t a_{1}-324 a_{2}{ }^{5} a_{1}+216 a_{2}{ }^{3} a_{1}{ }^{3}+648 a_{2}{ }^{3} a_{1}{ }^{3} t-36 a_{1}{ }^{5} a_{2}-108 a_{1}{ }^{5} t a_{2}\right) x^{3}$ $+9 a_{1} a_{2}\left(972 a_{2}{ }^{5} t a_{1}-648 a_{2}^{3} a_{1}^{3} t-108 a_{2}^{3} a_{1}^{3}-972 a_{2}^{3} a_{1}^{3} t^{2}+162 a_{1}^{5} t^{2} a_{2}+162 a_{2}^{5} a_{1}+18 a_{1}{ }^{5} a_{2}+108 a_{1}{ }^{5} t a_{2}+1458 a_{2} t^{2} a_{1}\right) x^{2}+9 a_{1} a_{2}\left(-108 a_{1} t^{3} a_{2}+\right.$ $216 a_{2}^{2} c_{2} a_{1}+72 d_{2} a_{1}{ }^{3}-432 a_{2}{ }^{3} a_{1}{ }^{3} t+648 a_{2}{ }^{3} a_{1}{ }^{3} t^{3}-24 a_{1}{ }^{2} d_{1} a_{2}+648 a_{2}{ }^{3} a_{1}{ }^{3} t^{2}+648 a_{2}{ }^{5} t a_{1}-72 a_{1}{ }^{3} c_{2}+72 d_{1} a_{2}{ }^{3}+24 a_{1}{ }^{2} c_{1} a_{2}-972 a_{2}{ }^{5} a_{1} t^{3}-72 a_{2}{ }^{3} c_{1}-$ $\left.972 a_{2}{ }^{5} t^{2} a_{1}+72 a_{1}{ }^{5} t a_{2}-216 a_{2}{ }^{2} d_{2} a_{1}-108 a_{1}{ }^{5} t^{2} a_{2}\right) x+9 a_{1} a_{2}\left(24 a_{2}{ }^{3} c_{1}+24 a_{1}{ }^{3} c_{2}+324 a_{2}{ }^{5} a_{1} t^{3}+540 a_{2}{ }^{3} a_{1}{ }^{3} t^{2}+216 a_{2}{ }^{3} a_{1}{ }^{3} t-216 a_{2}{ }^{3} a_{1}{ }^{3} t^{3}+8 a_{1}{ }^{2} d_{1} a_{2}-\right.$ $90 a_{1}^{5} t^{2} a_{2}-36 a_{1}^{5} t a_{2}+36 a_{1}^{5} t^{3} a_{2}+243 t^{4} a_{2}{ }^{5} a_{1}-162 t^{4} a_{2}{ }^{3} a_{1}{ }^{3}+27 a_{1}^{5} t^{4} a_{2}+72 a_{2}{ }^{2} d_{2} a_{1}-810 a_{2}{ }^{5} t^{2} a_{1}-324 a_{2}{ }^{5} t a_{1}-72 t a_{2}{ }^{3} d_{1}-72 a_{1}{ }^{3} t d_{2}+216 t a_{2}{ }^{2} d_{2} a_{1}+$ $\left.24 a_{1}^{2} t d_{1} a_{2}-216 t a_{2}^{2} c_{2} a_{1}-24 a_{1}^{2} t c_{1} a_{2}-24 d_{2} a_{1}^{3}-24 d_{1} a_{2}^{3}-8 a_{1}^{2} c_{1} a_{2}+72 t a_{2}{ }^{3} c_{1}+72 a_{1}{ }^{3} t c_{2}-72 a_{2}{ }^{2} c_{2} a_{1}\right)$,

and

$d(x, t)=\left(-9 a_{1}^{3} a_{2}+27 a_{1} a_{2}^{3}\right) x^{3}+\left(27 t a_{1}^{3} a_{2}-81 t a_{1} a_{2}^{3}+9 a_{1}^{3} a_{2}-27 a_{1} a_{2}^{3}\right) x^{2}+\left(-27 t^{2} a_{1}^{3} a_{2}+81 t^{2} a_{1} a_{2}{ }^{3}-18 t a_{1}^{3} a_{2}+54 t a_{1} a_{2}^{3}\right) x+9 t^{3} a_{1}^{3} a_{2}-$ $27 t^{3} a_{1} a_{2}{ }^{3}+9 t^{2} a_{1}^{3} a_{2}-27 t^{2} a_{1} a_{2}{ }^{3}+18 t a_{1}^{3} a_{2}-54 t a_{1} a_{2}{ }^{3}-12 a_{1} c_{2}+12 a_{1} d_{2}+4 a_{2} c_{1}-4 a_{2} d_{1}$,

is a rational solution to the Boussinesq equation (1.1), quotient of two polynomials with numerator of order 4 in $x$ and $t$, denominator of degree 6 in $x$ and $t$.
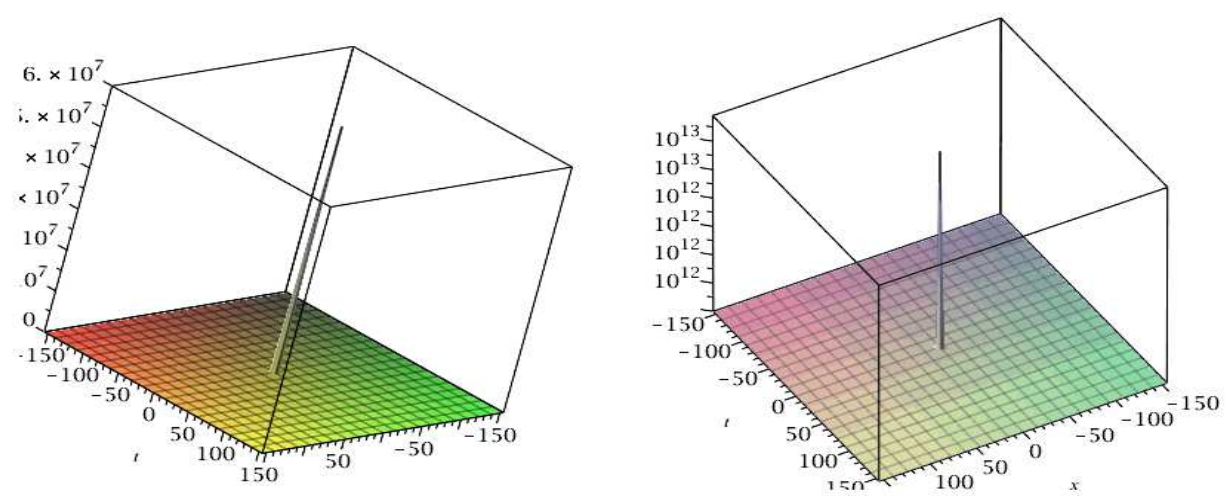

Figure 2.2: Solution of order 2 to (1.1); on the left, $a_{1}=10^{7}, a_{2}=1, c_{1}=1, c_{2}=1, d_{1}=0, d_{2}=0$; on the right, $a_{1}=1, a_{2}=10^{7}, c_{1}=1, c_{2}=1, d_{1}=0$, $d_{2}=0$. 

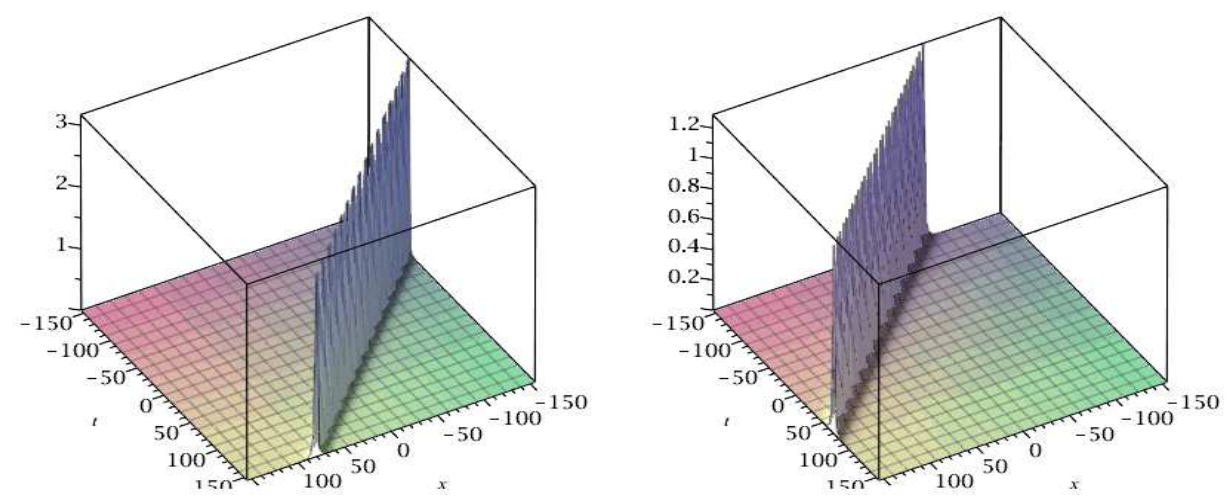

Figure 2.3: Solution of order 2 to (1.1); on the left, $a_{1}=1, a_{2}=1, c_{1}=10^{7}, c_{2}=0, d_{1}=1, d_{2}=1$; on the right, $a_{1}=1, a_{2}=1, c_{1}=0, c_{2}=10^{7}, d_{1}=0$, $d_{2}=0$.

\subsection{Rational solutions of order three}

We get the following rational solutions given by :

Theorem 2.9. The function $v$ defined by

$$
v(x, t)=-2 \frac{n(x, t)}{d(x, t)^{2}},
$$

is a rational solution to the Boussinesq equation (1.1), quotient of two polynomials with numerator of order 10 in $x$ and $t$, denominator of degree 12 in $x$ and $t$.

Because of the length of the solution, we give it only in the appendix.

Remark 2.10. If $c_{1}=c_{2}=c_{3}=d_{1}=d_{2}=d_{3}=0$, then the determinant in the formula (2.12) can be simplified by $\frac{(177147}{80} a_{1} a_{2} a_{3}^{3}\left(-80 a_{2}^{4}+\right.$ $\left.360 a_{3}^{2} a_{2}^{2}+a_{1}^{4}-30 a_{3}^{2} a_{1}^{2}\right)$ and the solution to the Boussinseq equation depends no longer on any parameter.

If one of the parameters $a_{1}, a_{2}$ or $a_{3}$ is equal to 0 then the solution of the Boussinesq equation is the trivial solution (equal to 0 ).
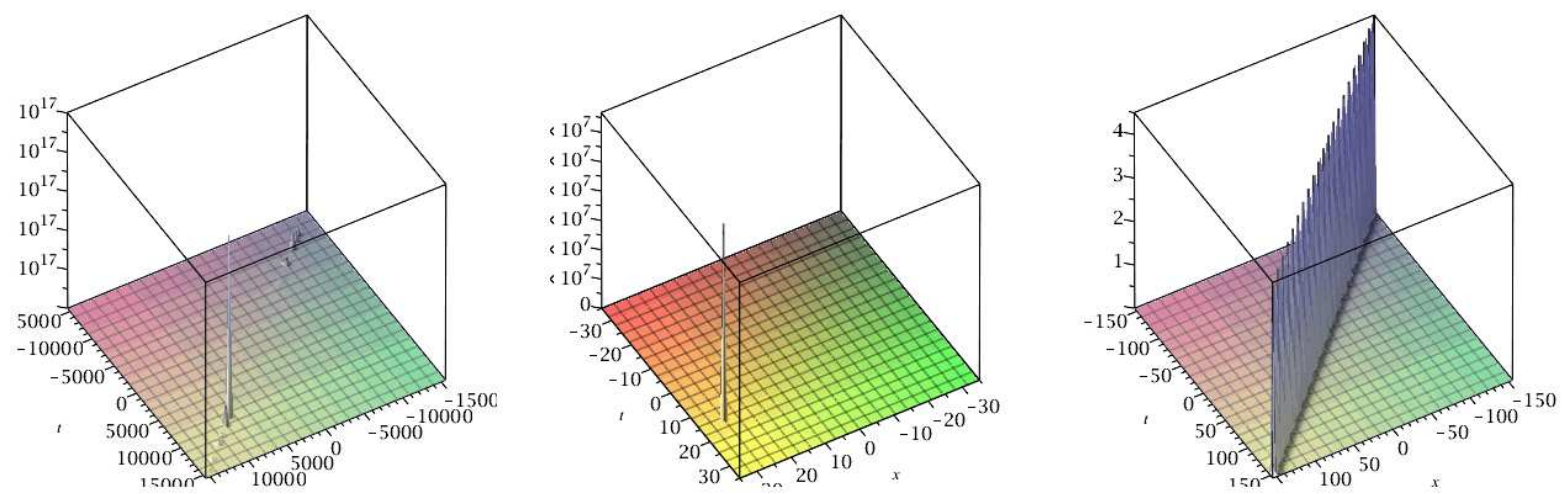

Figure 2.4: Solution of order 3 to (1.1); on the left, $a_{1}=1, a_{2}=1, a_{3}=1, c_{1}=0, c_{2}=0, c_{3}=10^{7}, d_{1}=0, d_{2}=0, d_{3}=0$; in the center, $a_{1}=1, a_{2}=1$, $a_{3}=1, c_{1}=0, c_{2}=10^{7}, c_{3}=1, d_{1}=0, d_{2}=0, d_{3}=0$; on the right, $a_{1}=1, a_{2}=1, a_{3}=10^{7}, c_{1}=1, c_{2}=1, c_{3}=1, d_{1}=0, d_{2}=0, d_{3}=0$.
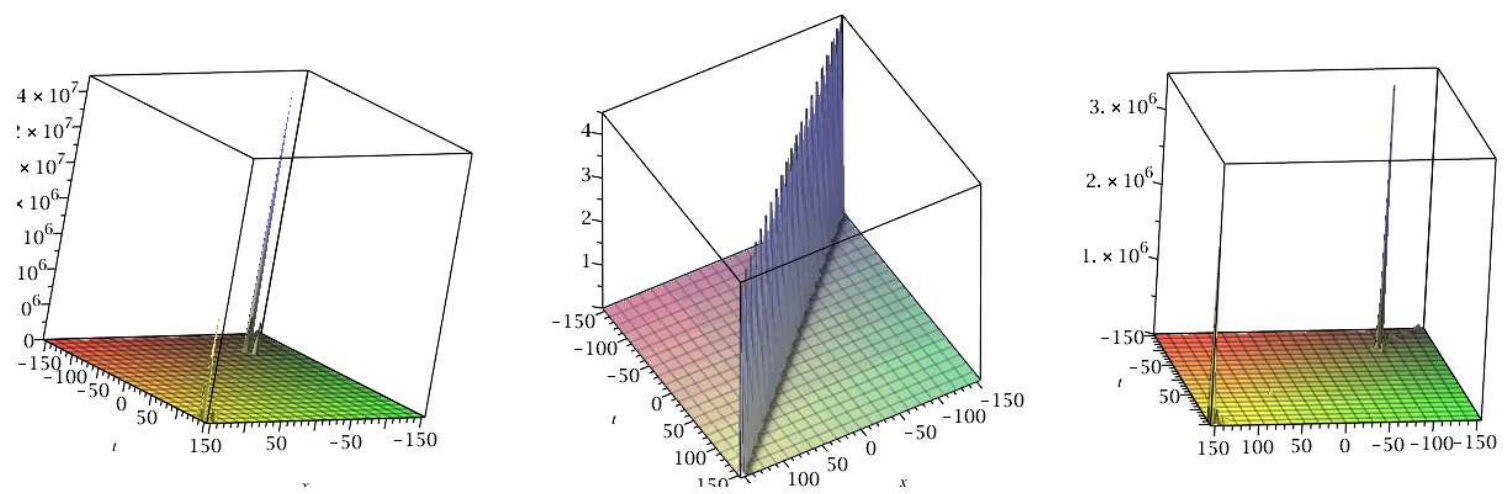

Figure 2.5: Solution of order 3 to (1.1)on the left, $a_{1}=1, a_{2}=10^{7}, a_{3}=1, c_{1}=1, c_{2}=1, c_{3}=10^{7}, d_{1}=0, d_{2}=0, d_{3}=0$; in the center, $a_{1}=10^{7}, a_{2}=1$, $a_{3}=1, c_{1}=1, c_{2}=10^{7}, c_{3}=1, d_{1}=0, d_{2}=0, d_{3}=0$; on the right, $a_{1}=1, a_{2}=1, a_{3}=10^{7}, c_{1}=10^{5}, c_{2}=1, c_{3}=1, d_{1}=0, d_{2}=0, d_{3}=0$. 


\section{Solutions to the Kadomtsev Petviashvili equation}

We consider the Kadomtsev Petviashvili equation (KP) which can be written in the form

$$
\left(4 u_{T}-6 u u_{X}+u_{X X X}\right)_{X}-3 u_{Y Y}=0,
$$

where subscripts $X, Y$ and $T$ denote as usual partial derivatives.

From the previous study, we can deduce easily solutions to the KP equation. It is sufficient for this, to use the following transformations $x={ }_{1} X+\frac{31 T}{4}, t={ }_{1} Y$ from the solutions to the Boussinesq equation to obtain solutions to the KP equation.

\subsection{Solutions to the KP equation}

\subsection{First order rational solutions}

We have the following result at order $N=1$ :

Theorem 3.1. The function $v$ defined by

$$
v(X . Y, T)=\frac{-288 a_{1}^{2}}{\left(12 i a_{1} X+9 i a_{1} T+4 c_{1}-12 i Y a_{1}-4 d_{1}\right)^{2}},
$$

is a solution to the KP equation (3.1).

Remark 3.2. The solution (3.2) can be simplified and be rewritten as depending on one parameter $v(X . Y, T)=\frac{-288}{\left(12 i X+9 i T+4 C_{1}-12 i Y\right)^{2}}$
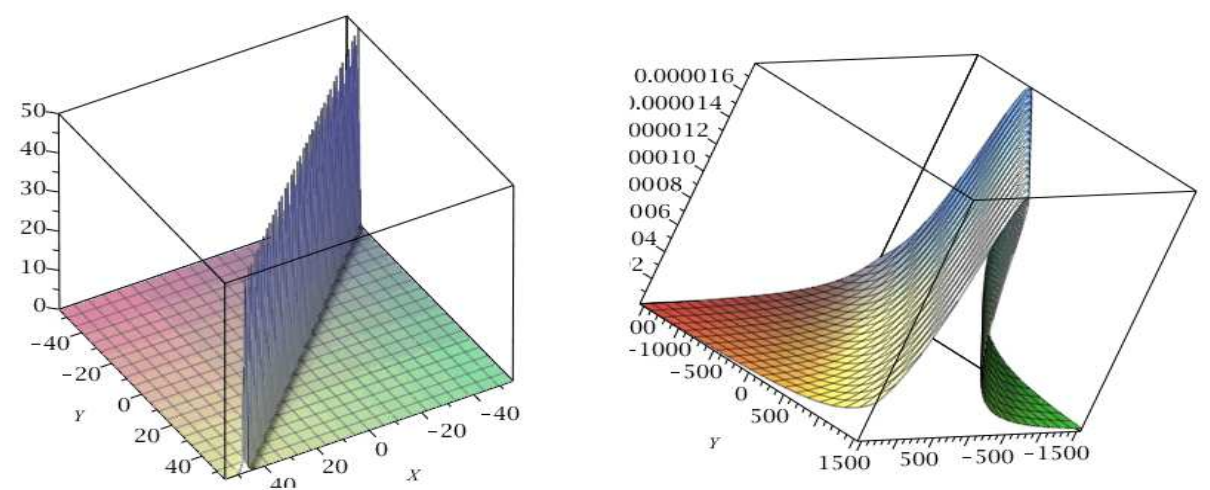

Figure 3.1: Solution of order 1 to (3.1), on the left $T=10, a_{1}=10^{6}, c_{1}=1, d_{1}=1$; on the right $T=10, a_{1}=1, c_{1}=10^{3}, d_{1}=1$.

Remark 3.3. The case where $T=10, a_{1}=1, c_{1}=1, d_{1}=10^{3}$ gives the same figure as the case $T=10, a_{1}=1, c_{1}=10^{3}, d_{1}=1$.

\subsection{Second order rational solutions}

We obtain the following solutions :

Theorem 3.4. The function $v$ defined by

$$
v(X . Y, T)=-2 \frac{n(X . Y, T)}{d(X . Y, T)^{2}}
$$

with

$n(X . Y, T)=144 a_{1} a_{2}\left(-41472 a_{2}{ }^{3} a_{1}{ }^{3}+62208 a_{2}{ }^{5} a_{1}+6912 a_{1}{ }^{5} a_{2}\right) X^{4}+144 a_{1} a_{2}\left(-124416 a_{2}{ }^{3} a_{1}{ }^{3} T-27648 a_{1}{ }^{5} Y a_{2}+165888 a_{2}{ }^{3} a_{1}{ }^{3} Y+9216 i a_{1}{ }^{5} a_{2}\right.$ $\left.-55296 i a_{2}{ }^{3} a_{1}{ }^{3}+186624 a_{2}{ }^{5} a_{1} T+82944 i a_{2}{ }^{5} a_{1}+20736 a_{1}{ }^{5} a_{2} T-248832 a_{2}{ }^{5} Y a_{1}\right) X^{3}+144 a_{1} a_{2}\left(-248832 a_{2}{ }^{3} a_{1}{ }^{3} Y^{2}-27648 i a_{1}{ }^{5} Y a_{2}+23328 a_{1}{ }^{5} a_{2} T^{2}+\right.$ $373248 a_{2}{ }^{3} a_{1}{ }^{3} Y T+165888 i a_{2}{ }^{3} a_{1}{ }^{3} Y-124416 i a_{2}{ }^{3} a_{1}{ }^{3} T+41472 a_{1}{ }^{5} Y^{2} a_{2}-62208 a_{1}{ }^{5} Y a_{2} T-559872 a_{2}{ }^{5} Y a_{1} T-139968 a_{2}{ }^{3} a_{1}{ }^{3} T^{2}-41472 a_{2}{ }^{5} a_{1}+27648 a_{2}{ }^{3} a_{1}{ }^{3}+$ $\left.209952 a_{2}{ }^{5} a_{1} T^{2}+186624 i a_{2}{ }^{5} a_{1} T+373248 a_{2}{ }^{5} Y^{2} a_{1}-248832 i a_{2}{ }^{5} Y a_{1}+20736 i a_{1}{ }^{5} a_{2} T-4608 a_{1}{ }^{5} a_{2}\right) X^{2}+144 a_{1} a_{2}\left(-419904 a_{2}{ }^{5} Y a_{1} T^{2}+279936 a_{2}{ }^{3} a_{1}{ }^{3} Y T^{2}-\right.$ $46656 a_{1}{ }^{5} Y a_{2} T^{2}+62208 a_{1}{ }^{5} Y^{2} a_{2} T+559872 a_{2}{ }^{5} Y^{2} a_{1} T-373248 a_{2}{ }^{3} a_{1}{ }^{3} Y^{2} T-373248 i a_{2}{ }^{5} Y a_{1} T+248832 i a_{2}{ }^{3} a_{1}{ }^{3} Y T-41472 i a_{1}{ }^{5} Y a_{2} T-18432 i a_{2}{ }^{3} c_{1}+$ $41472 a_{2}{ }^{3} a_{1}{ }^{3} T-6912 a_{1}{ }^{5} a_{2} T-62208 a_{2}{ }^{5} a_{1} T+18432 i d_{2} a_{1}{ }^{3}-18432 i a_{1}{ }^{3} c_{2}+18432 i d_{1} a_{2}{ }^{3}+139968 i a_{2}{ }^{5} a_{1} T^{2}-93312 i a_{2}{ }^{3} a_{1}{ }^{3} T^{2}+15552 i a_{1}{ }^{5} a_{2} T^{2}+$ $27648 i a_{1}{ }^{5} Y^{2} a_{2}+248832 i a_{2}{ }^{5} Y^{2} a_{1}-165888 i a_{2}{ }^{3} a_{1}{ }^{3} Y^{2}-55296 i a_{2}^{2} d_{2} a_{1}-6144 i a_{1}{ }^{2} d_{1} a_{2}+55296 i a_{2}{ }^{2} c_{2} a_{1}+6144 i a_{1}{ }^{2} c_{1} a_{2}-69984 a_{2}{ }^{3} a_{1}{ }^{3} T^{3}+11664 a_{1}{ }^{5} a_{2} T^{3}+$ $\left.104976 a_{2}^{5} a_{1} T^{3}-248832 a_{2}{ }^{5} a_{1} Y^{3}+110592 a_{2}{ }^{3} a_{1}{ }^{3} Y+165888 a_{2}{ }^{3} a_{1}{ }^{3} Y^{3}-18432 a_{1}{ }^{5} Y a_{2}-27648 a_{1}{ }^{5} Y^{3} a_{2}-165888 a_{2}{ }^{5} Y a_{1}\right) X+144 a_{1} a_{2}\left(6144 a_{2}{ }^{3} c_{1}+\right.$ $6144 a_{1}{ }^{3} c_{2}+41472 i a_{2}{ }^{2} c_{2} a_{1} T+4608 i a_{1}{ }^{2} c_{1} a_{2} T-104976 a_{2}{ }^{5} Y a_{1} T^{3}+69984 a_{2}{ }^{3} a_{1}{ }^{3} Y T^{3}-11664 a_{1}{ }^{5} Y a_{2} T^{3}+55296 i Y a_{2}{ }^{2} d_{2} a_{1}+6144 i a_{1}{ }^{2} Y d_{1} a_{2}$ $-55296 i Y a_{2}{ }^{2} c_{2} a_{1}-6144 i a_{1}{ }^{2} Y c_{1} a_{2}-139968 i a_{2}{ }^{5} Y a_{1} T^{2}+93312 i a_{2}{ }^{3} a_{1}{ }^{3} Y T^{2}-15552 i a_{1}{ }^{5} Y a_{2} T^{2}+20736 i a_{1}{ }^{5} Y^{2} a_{2} T+186624 i a_{2}{ }^{5} Y^{2} a_{1} T-124416 i a_{2}{ }^{3} a_{1}{ }^{3} Y^{2} T-$ $41472 i a_{2}{ }^{2} d_{2} a_{1} T-4608 i a_{1}{ }^{2} d_{1} a_{2} T-13122 a_{2}{ }^{3} a_{1}{ }^{3} T^{4}+19683 a_{2}{ }^{5} a_{1} T^{4}+2187 a_{1}{ }^{5} a_{2} T^{4}+13824 i d_{2} a_{1}{ }^{3} T-13824 i a_{1}{ }^{3} c_{2} T+13824 i d_{1} a_{2}{ }^{3} T-23328 i a_{2}{ }^{3} a_{1}{ }^{3} T^{3}+$ $3888 i a_{1}{ }^{5} a_{2} T^{3}-82944 i a_{2}{ }^{5} a_{1} Y^{3}+55296 i a_{2}{ }^{3} a_{1}{ }^{3} Y+55296 i a_{2}{ }^{3} a_{1}{ }^{3} Y^{3}-9216 i a_{1}{ }^{5} Y a_{2}-9216 i a_{1}{ }^{5} Y^{3} a_{2}-82944 i a_{2}{ }^{5} Y a_{1}-18432 i Y a_{2}{ }^{3} d_{1}-18432 i a_{1}{ }^{3} Y d_{2}+$ $18432 i Y a_{2}{ }^{3} c_{1}+18432 i a_{1}{ }^{3} Y c_{2}+34992 i a_{2}{ }^{5} a_{1} T^{3}-13824 i a_{2}{ }^{3} c_{1} T-124416 a_{2}{ }^{5} Y a_{1} T+82944 a_{2}{ }^{3} a_{1}{ }^{3} Y T-186624 a_{2}{ }^{5} a_{1} Y^{3} T+124416 a_{2}{ }^{3} a_{1}{ }^{3} Y^{3} T-13824 a_{1}{ }^{5} Y a_{2} T$ 
$-20736 a_{1}^{5} Y^{3} a_{2} T+209952 a_{2}{ }^{5} Y^{2} a_{1} T^{2}-139968 a_{2}{ }^{3} a_{1}{ }^{3} Y^{2} T^{2}+23328 a_{1}{ }^{5} Y^{2} a_{2} T^{2}+62208 Y^{4} a_{2}{ }^{5} a_{1}-41472 Y^{4} a_{2}{ }^{3} a_{1}{ }^{3}+6912 a_{1}{ }^{5} Y^{4} a_{2}+2048 a_{1}{ }^{2} d_{1} a_{2}+$ $18432 a_{2}{ }^{2} d_{2} a_{1}-6144 d_{2} a_{1}{ }^{3}-6144 d_{1} a_{2}{ }^{3}+15552 a_{2}{ }^{3} a_{1}{ }^{3} T^{2}-2592 a_{1}{ }^{5} a_{2} T^{2}-23328 a_{2}{ }^{5} a_{1} T^{2}-138240 a_{2}{ }^{3} a_{1}{ }^{3} Y^{2}+207360 a_{2}{ }^{5} Y^{2} a_{1}+23040 a_{1}{ }^{5} Y^{2} a_{2}-$ $\left.2048 a_{1}^{2} c_{1} a_{2}-18432 a_{2}^{2} c_{2} a_{1}\right)$,

and

$d(X . Y, T)=-1728 i a_{1} a_{2}{ }^{3} X^{3}+576 i a_{1}{ }^{3} a_{2} X^{3}+1728 a_{1} a_{2}{ }^{3} X^{2}-576 a_{1}{ }^{3} a_{2} X^{2}-3888 i a_{1} a_{2}{ }^{3} X^{2} T+5184 i Y a_{1} a_{2}{ }^{3} X^{2}-1728 i Y a_{1}{ }^{3} a_{2} X^{2}+1296 i a_{1}{ }^{3} a_{2} T X^{2}+$ $7776 i Y a_{1} a_{2}{ }^{3} T X+972 i a_{1}{ }^{3} a_{2} T^{2} X-2916 i a_{1} a_{2}{ }^{3} X T^{2}-5184 i Y^{2} a_{1} a_{2}{ }^{3} X+1728 i Y^{2} a_{1}{ }^{3} a_{2} X-2592 i Y a_{1}{ }^{3} a_{2} X T-864 a_{1}{ }^{3} a_{2} T X-3456 Y a_{1} a_{2}{ }^{3} X+2592 a_{1} a_{2}{ }^{3} T X+$ $1152 Y a_{1}{ }^{3} a_{2} X-768 a_{1} c_{2}-256 a_{2} d_{1}-3888 i Y^{2} a_{1} a_{2}{ }^{3} T+2916 i Y a_{1} a_{2}{ }^{3} T^{2}+1728 i Y^{3} a_{1} a_{2}{ }^{3}-576 i Y^{3} a_{1}{ }^{3} a_{2}-3456 i Y a_{1} a_{2}{ }^{3}+1152 i Y a_{1}{ }^{3} a_{2}-972 i Y a_{1}{ }^{3} a_{2} T^{2}-$ $729 i a_{1} a_{2}{ }^{3} T^{3}+256 a_{2} c_{1}+243 i a_{1}{ }^{3} a_{2} T^{3}+768 a_{1} d_{2}+1296 i Y^{2} a_{1}{ }^{3} a_{2} T+972 a_{1} a_{2}{ }^{3} T^{2}-2592 Y a_{1} a_{2}{ }^{3} T-324 a_{1}{ }^{3} a_{2} T^{2}+864 Y a_{1}{ }^{3} a_{2} T+1728 Y^{2} a_{1} a_{2}{ }^{3}-576 Y^{2} a_{1}{ }^{3} a_{2}$, is a rational solution to the KP equation (3.1), quotient of two polynomials with numerator of degree 4 in $x$, $y$ and t and denominator of degree 6 in $x, y$ and $t$.
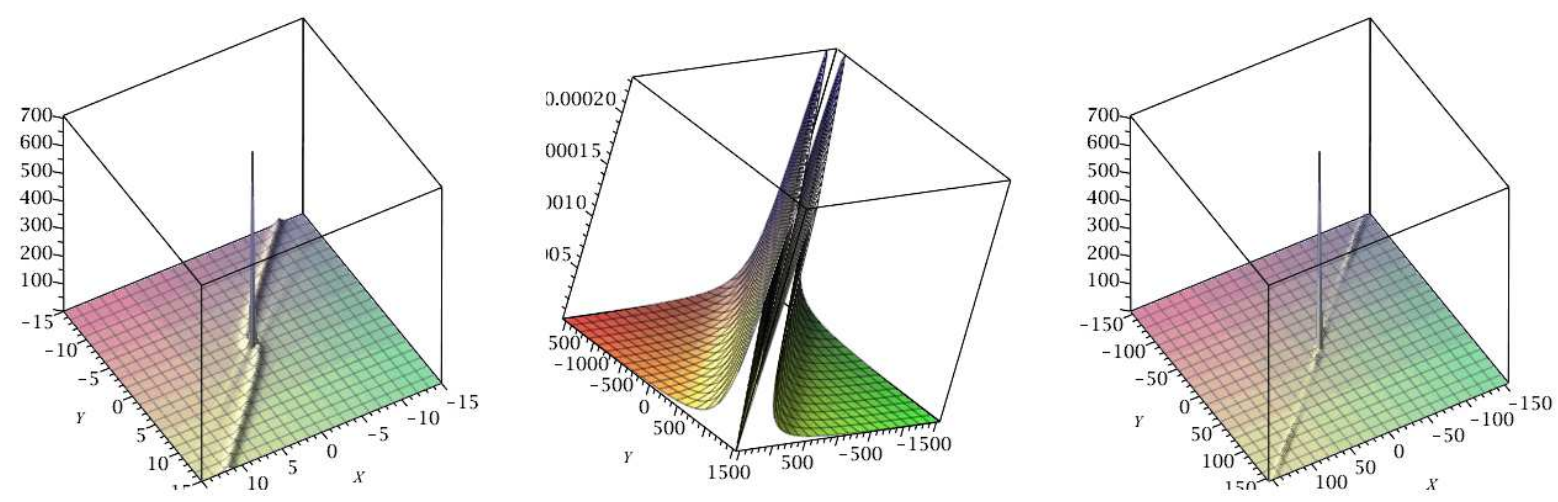

Figure 3.2: Solution of order 2 to (3.1); on the left $T=0,1, a_{1}=1, a_{2}=1, c_{1}=0, c_{2}=0, d_{1}=0, d_{2}=0$; in the center $T=0,1, a_{1}=1, a_{2}=1, c_{1}=0$, $c_{2}=10^{8}, d_{1}=0, d_{2}=0$; on the right $T=0,1, a_{1}=1, a_{1}=10^{9}, c_{1}=1, c_{2}=1, d_{1}=0, d_{2}=0$.
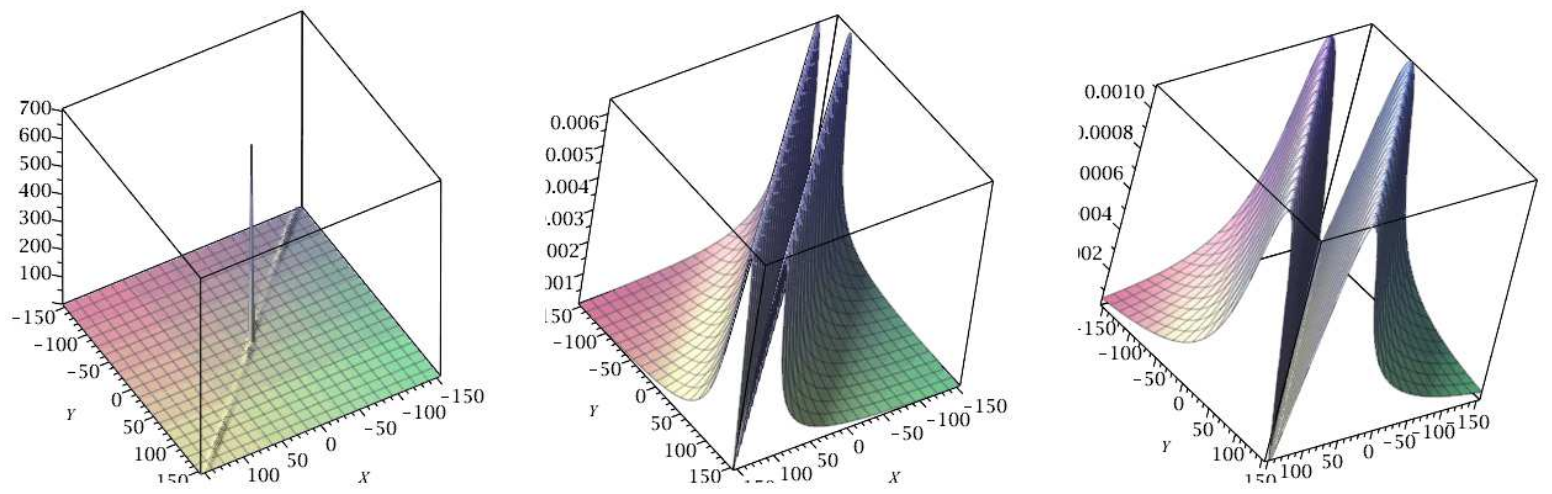

Figure 3.3: Solution of order 2 to (3.1); on the left $T=0,1, a_{1}=10^{6}, a_{2}=1, c_{1}=1, c_{2}=1, d_{1}=0, d_{2}=0$; in the center $T=0,1, a_{1}=1, a_{2}=1, c_{1}=10^{6}$, $c_{2}=0, d_{1}=0, d_{2}=0$; on the right $T=10, a_{1}=1, a_{2}=1, c_{1}=1, c_{2}=10^{7}, d_{1}=0, d_{2}=0$.
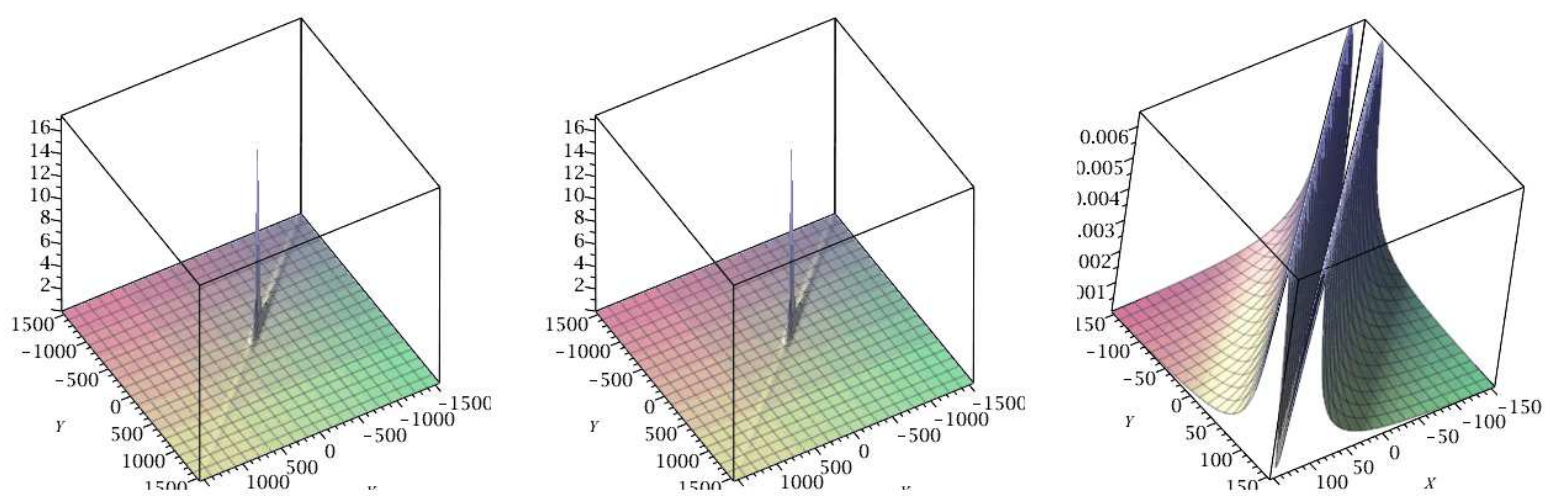

Figure 3.4: Solution of order 2 to (3.1); on the left $T=10, a_{1}=1, a_{2}=10^{9}, c_{1}=1, c_{2}=1, d_{1}=0, d_{2}=0$; in the center $T=10, a_{1}=10^{10}, a_{2}=1, c_{1}=1$, $c_{2}=1, d_{1}=0, d_{2}=0$; on the right $T=10, a_{1}=1, a_{2}=1, c_{1}=1, c_{2}=10^{6}, d_{1}=0, d_{2}=0$. 


\subsection{Rational solutions of order 3}

We get the non singular rational solutions given by :

Theorem 3.5. The function $v$ defined by

$$
v(X . Y, T)=-2 \frac{n(X . Y, T)}{d(X . Y, T)^{2}},
$$

is a rational solution to the KP equation (3.1), quotient of two polynomials with numerator of degree 10 in $X, Y$, $T$ and denominator of degree 12 in $X, Y$ and $T$.

Because of the length of the solution, we only give it in the appendix.
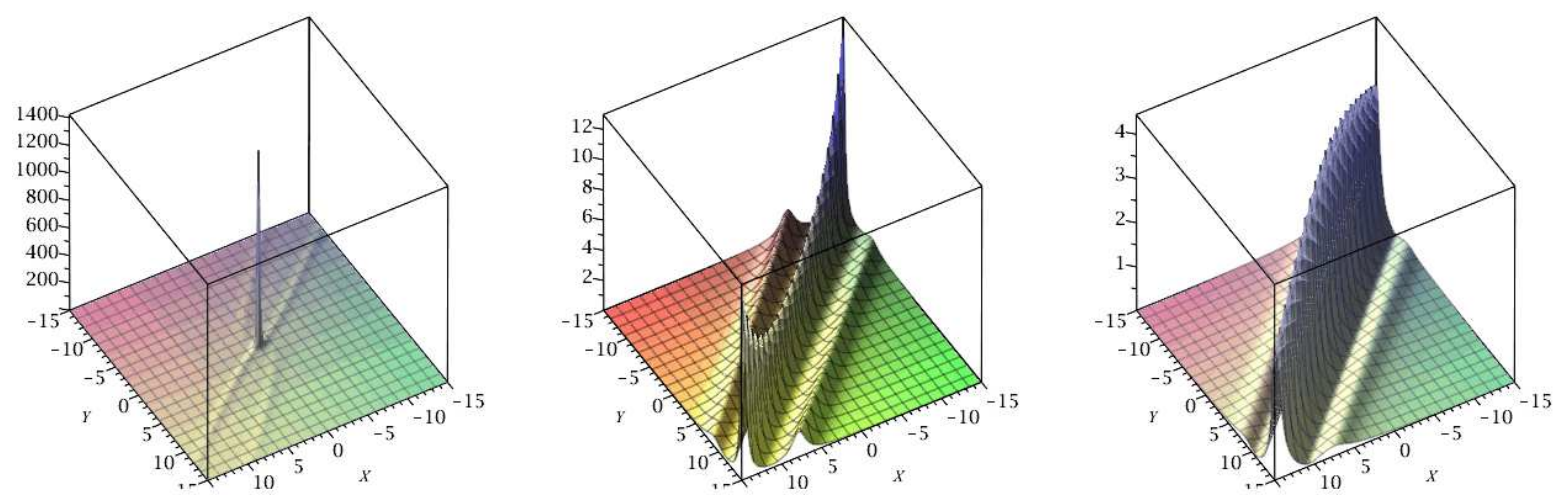

Figure 3.5: Solution of order 3 to (3.1); on the left $T=0,1, a_{1}=1, a_{2}=1, a_{3}=1, c_{1}=0, c_{2}=0 c_{3}=0, d_{1}=0, d_{2}=0, d_{3}=0$; in the center $T=0,1$, $a_{1}=1, a_{2}=1, a_{3}=1, c_{1}=1, c_{2}=0, c_{3}=10^{6}, d_{1}=0, d_{2}=0, d_{3}=0$; on the right $T=0,1, a_{1}=1, a_{2}=1, a_{3}=1, c_{1}=0, c_{2}=10^{6}, c_{3}=1, d_{1}=0$, $d_{2}=0, d_{3}=0$.
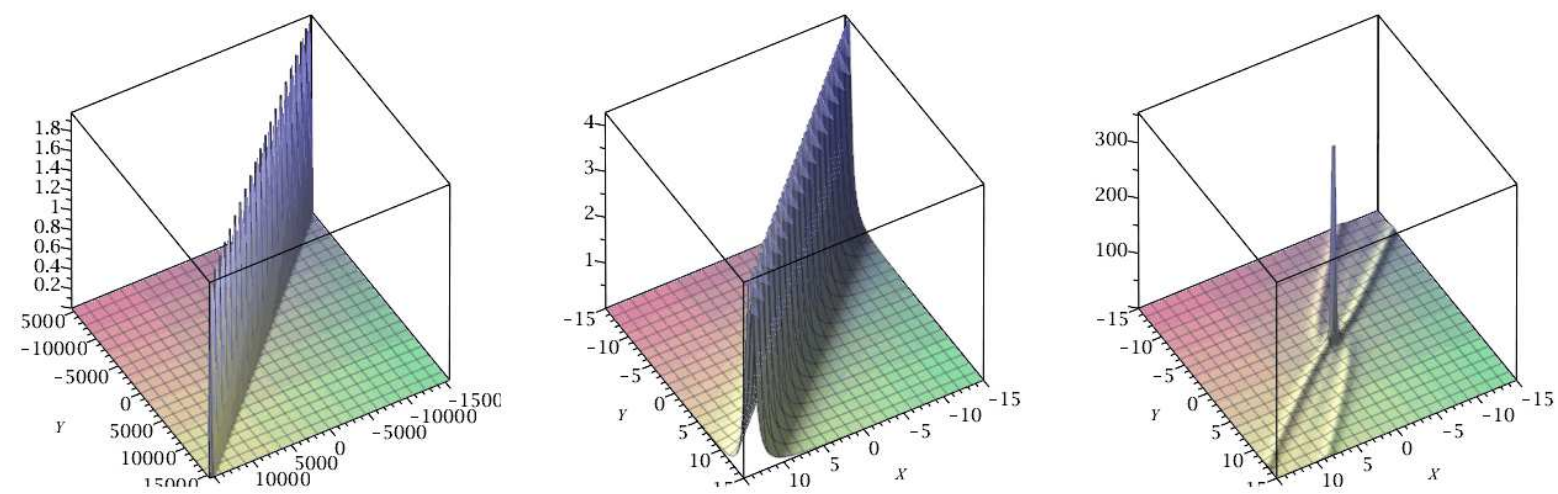

Figure 3.6: Solution of order 3 to (3.1); on the left $T=0,1, a_{1}=1, a_{2}=1, a_{3}=10^{24}, c_{1}=1, c_{2}=1 c_{3}=1, d_{1}=0, d_{2}=0, d_{3}=0$; in the center $T=0,1$, $a_{1}=1, a_{2}=10^{4}, a_{3}=1, c_{1}=1, c_{2}=1, c_{3}=1, d_{1}=0, d_{2}=0, d_{3}=0$; on the right $T=0,1, a_{1}=10, a_{2}=1, a_{3}=1, c_{1}=1, c_{2}=10, c_{3}=1, d_{1}=0$, $d_{2}=0, d_{3}=0$.
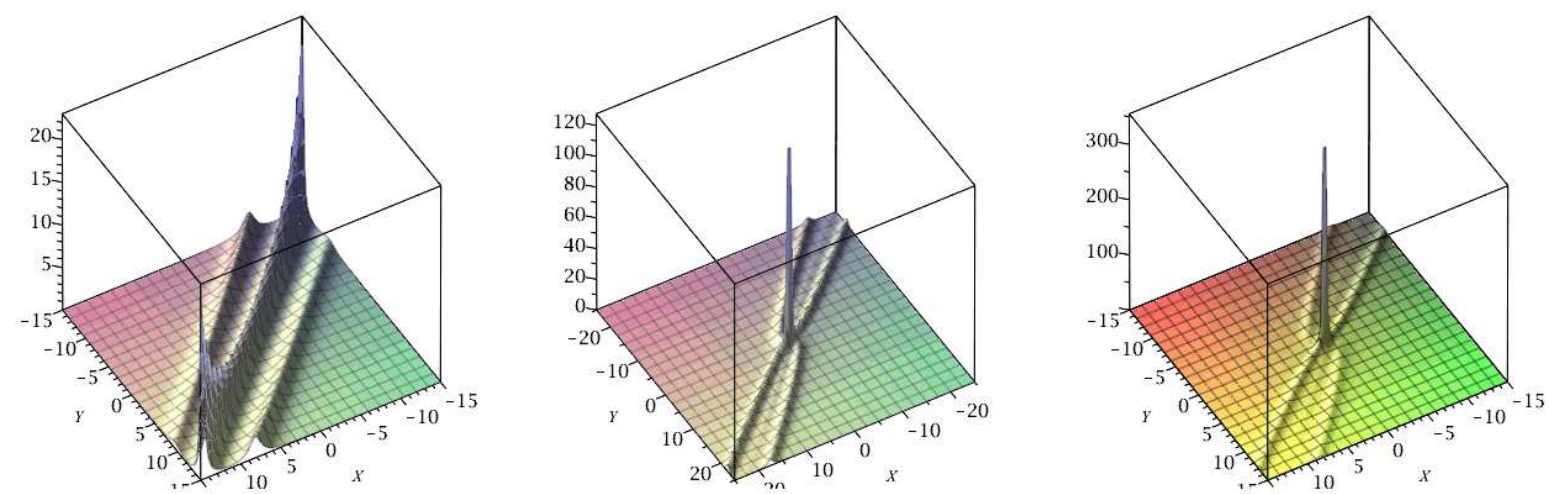

Figure 3.7: Solution of order 3 to (3.1); on the left $T=0,1, a_{1}=1, a_{2}=1, a_{3}=1, c_{1}=10^{6}, c_{2}=1 c_{3}=1, d_{1}=0, d_{2}=0, d_{3}=0$; in the center $T=1$, $a_{1}=1, a_{2}=1, a_{3}=1, c_{1}=0, c_{2}=0, c_{3}=0, d_{1}=0, d_{2}=0, d_{3}=0$; on the right $T=1, a_{1}=10^{6}, a_{2}=1, a_{3}=1, c_{1}=1, c_{2}=1, c_{3}=1, d_{1}=0, d_{2}=0$, $d_{3}=0$. 

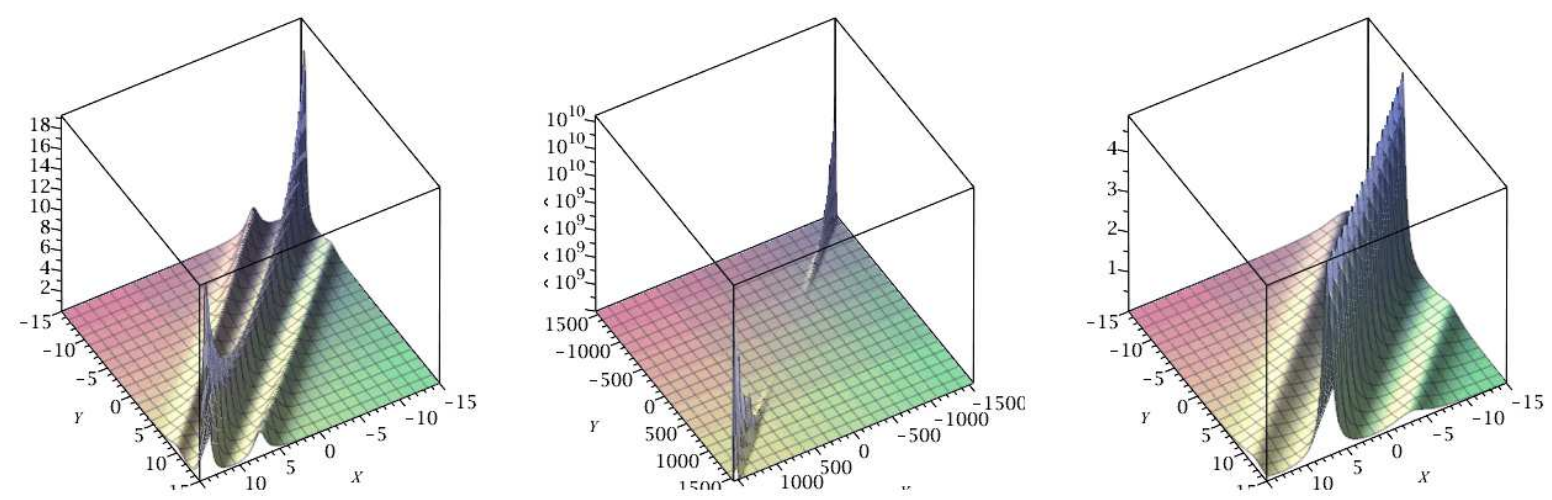

Figure 3.8: Solution of order 3 to (3.1); on the left $T=1, a_{1}=1, a_{2}=1, a_{3}=1, c_{1}=10^{6}, c_{2}=1 c_{3}=1, d_{1}=0, d_{2}=0, d_{3}=0$; in the center $T=10$, $a_{1}=10^{6}, a_{2}=1, a_{3}=1, c_{1}=1, c_{2}=1, c_{3}=1, d_{1}=0, d_{2}=0, d_{3}=0$; on the right $T=10, a_{1}=1, a_{2}=1, a_{3}=1, c_{1}=1, c_{2}=1, c_{3}=10^{7}, d_{1}=0, d_{2}=0$, $d_{3}=0$.

\section{Conclusion}

We have given three types of representations of solutions to the Boussinesq equation. First, solutions in terms of elementary exponential functions have been constructed. In particular, performing a passage to the limit when one parameter goes to 0 we get rational solutions to the Boussinesq equation. We give an other representation in terms of determinants without the presence of a limit. So we obtain an infinite hierarchy of multiparametric families of rational solutions to the Boussinesq equation as a quotient of a polynomial of degree $N(N+1)-2$ in $x, t$ by a polynomial of degree $N(N+1)$ in $x, t$ depending on $3 N$ real parameters.

As a byproduct, we get easily similar rational solutions to the Kadomtsev Petviashvili equation as the quotient of determinants of polynomials, where the numerator is a polynomial of degree $N(N+1)-2$ in $X, Y, T$ and the denominator is a polynomial of degree $N(N+1)$ in $X, Y, T$. In particular, we construct explicit rational solutions to the Boussinesq equation of order 1,2, 3 .

Unlike other equations such as NLS, there are no specific structures that emerge as a function of the parameters.

\section{References}

[1] J. Boussinesq, Théorie de l'intumescence appelée onde solitaire ou de translation se propageant dans un canal rectangulaire, C.R.A.S., V 72 (1871), $755-759$

[2] J. Boussinesq, Théorie des ondes et des remous qui se propagent le long d'un canal rectangulaire horizontal, en communiquant au liquide contenu dans ce canal des vitesses sensiblement pareilles de la surface au fond, J. Math. Pures Appl., Ser. 2, T. 17, (1872), 55-108

[3] M.J. Ablowitz, P.A. Clarkson, Solitons, Nonlinear Evolution Equations and Inverse Scattering, L.M.S. Lect. Notes Math., (1991) 149

[4] P. Deift, C. Tomei, E. Trubowitz, Inverse scattering and the Boussinesq equation, Com. Pure Appl. Math., 35, (1992), 567-628

[5] M. Toda,Studies of a nonlinear lattice, Phys. Rep., 8, (1975), 1-125

[6] V.E. Zakharov, On stochastization of one-dimensional chains of nonlinear oscillations, Sov. Phys. JETP, 38, (1974), 108-110

[7] E. Infeld, G. Rowlands, Nonlinear Waves, Solitons and Chaos, C.U.P., 1990

[8] R. Hirota, J. Satsuma, Non linear evolution equations generated from the Bäcklund transformation fot the Boussinesq equation, Prog. of Theor. Phys. 57, (1977), 797-807

[9] M.J. Ablowitz, J. Satsuma, Solitons and rational solutions of nonlinear evolution equations, J. Math. Phys., 19, (1978), 2180-2186

[10] J.J.C. Nimmo, N.C. Freemann, A method of obtaining the N soliton solution of the Boussinesq equation in terms of a wronskian, Phys. Lett., 95, N. 1, (1983), 4-6

[11] V.B. Matveev, A.O. Smirnov, On the Riemann theta function of a trigonal curve and solutions of the Boussinesq anf KP equations, L.M.P., 14, (1987),

[12] V.B. Matveev and M.A. Salle, Darboux transformations and solitons, Series in Nonlinear Dynamics, Springer-Verlag, Berlin, 1991

[13] L.V. Bogdanov, V.E. Zakharov, The Boussinesq equation revisited, Phys. D, 165, (2002), 137-162

[14] P.A. Clarkson, Rational solutions of the Boussinesq equation, Anal. Appl., 6, (2006), 349-369

[15] P.A. Clarkson, Rational solutions of the classical Boussinesq system, Nonlin. Anal. : Real World Appl., 10, (2010), 3361-3371

[16] P.A. Clarkson, E. Dowie, Rational solutions of the Boussinesq equation and applications to rogue waves, Trans. of Math. and its Appl., 1, (2017), 1-26 